\title{
ORIGINAL ARTICLE \\ Kiwifruit defense protein, kiwellin (Act d 5) percutaneously sensitizes mouse models through the epidermal application of crude kiwifruit extract
}

\author{
Serina Kinugasa', Shota Hidaka', Serina Tanaka', Eri Izumi', Nobuhiro Zaima',2 and Tatsuya \\ Moriyama ${ }^{1,2 *}$ \\ 'Department of Applied Biological Chemistry, Graduate School of Agriculture, Kindai University, Nara, Japan; \\ ${ }^{2}$ Agricultural Technology and Innovation Research Institute, Kindai University, Nara, Japan
}

\section{Popular scientific summary}

- In a mouse model, specific IgE and IgG1 antibodies were produced by percutaneous application of crude extracts from green and gold kiwifruit.

- These antibodies reacted with 18, 23, and $24 \mathrm{kDa}$ proteins in both green and gold kiwifruits.

- The amino acid sequences of these three novel proteins partially matched with that of kiwellin (Act d 5), indicating that they could be percutaneously sensitizing kiwifruit allergens.

\section{Abstract}

Background: Kiwifruit is a popular fruit consumed worldwide and is also used as a cosmetic ingredient. However, it is known to cause allergic reactions in humans. Recent studies have suggested an association between food allergy and food allergens entering the body via the skin. However, percutaneously sensitizing kiwifruit allergens have not been identified in human studies or in animal models.

Objective: This study aimed to identify kiwifruit proteins that percutaneously sensitized mice through the epidermal application of crude extracts from green and gold kiwifruit on the dorsal skin, and serum IgE and IgG1 levels were used as sensitization markers.

Design: BALB/c mice were back-shaved and their skin was exposed to crude extracts from green and gold kiwifruit that contained sodium dodecyl sulfate. Specific IgE and IgG1 antibodies generated and secreted in response to antigens were measured using enzyme-linked immunosorbent assay or immunoblotting.

Results: Skin exposure to kiwifruit extract induced an increase in the levels of kiwifruit-specific $\operatorname{IgE}$ and $\operatorname{IgG} 1$, which are helper $\mathrm{T}$ cell 2-related allergenic antibodies in mice. These antibodies reacted with 18, 23, and 24 $\mathrm{kDa}$ proteins found in both green and gold kiwifruits. Thus, three percutaneously sensitizing allergens were identified and purified. Their amino acid sequences partially matched with that of kiwellin (Act d 5).

Discussion and conclusion: Kiwellin has been identified as a plant defense-related protein. Interestingly, many plant allergens are biodefense-related proteins belonging to the pathogenesis-related protein family. Kiwellin, which was discovered to be a transdermal sensitizing antigen, might also be categorized as a biodefense-related protein. This study is the first to identify kiwellin (Act d 5) as a percutaneously sensitizing kiwifruit allergen in a mouse model.

Keywords: kiwifruits; allergens; kiwellin; Act d 5; allergenicity; percutaneous sensitization

Received: 9 February 2021; Revised: 22 August 2021; Accepted: 26 August 202 I; Published: 15 October 2021

$\mathrm{T}$ he prevalence of food allergies that disrupt the quality of life is increasing. Food allergies affect almost $5 \%$ adults and $8 \%$ children in developed countries (1). The gastrointestinal tract is equipped with an immune system that uses oral tolerance to prevent allergic reactions to food proteins
(2). However, abnormal functioning of this tolerance mechanism results in food allergy. In IgE-mediated food allergies, food allergens entering the body stimulate the immune system to produce allergen-specific IgE that binds to Fce receptors on mast cells, leading to sensitization (3). 
A recent study reported an association between food allergy and food allergens entering through the skin (4). Hydrolyzed wheat protein-containing soaps have been reported to induce wheat allergy (anaphylaxis) (5). Percutaneous application of soybean can also induce food allergies (6). Another study reported food allergies that preceded contact urticaria in response to contact with foods such as rice, wheat, fruits, vegetables, fish, shrimp, and cattle fish (7). Thus, many cases of food allergy occur because of the percutaneous entry of food allergens, followed by sensitization. Damage to the barrier function of the skin and inflammation cause percutaneous sensitization (8).

Previously, we identified that principal soybean allergens Gly $\mathrm{m} 5$ and Gly $\mathrm{m} 6$ trigger percutaneous sensitization in a mouse model (9). Using the same model, the soybean trypsin inhibitor Gly m TI was also identified as a soybean allergen that causes percutaneous sensitization (10). These food allergens are also the primary soybean allergens in humans, suggesting that a mouse model can be effectively used for the identification of allergens that cause percutaneous sensitization in humans.

Kiwifruit is rich in vitamin C, polyphenols (11), and dietary fiber, and has also been suggested to be effective in preventing heart disease and cancer (12). Green kiwifruit appeared in New Zealand in 1904, in the United States in 1962, and then in European countries (13). Gold kiwifruit appeared in the international market in 1999 (14). Thus, kiwifruit is a popular fruit consumed globally. However, kiwifruit is associated with high risk of allergenicity that was first reported in 1981 (15). Green and gold kiwifruits exhibit cross-reactivity. At present, 13 allergens have been identified in green kiwifruit and three allergens in gold kiwifruit. Among them, thiol protease $30 \mathrm{kDa}$ actinidin (Act d 1), $24 \mathrm{kDa}$ thaumatin-like protein (Act d 2), 17 kDa PR-10 (Act d 8), 17 kDa MLP/RRP (Act d 11), and $50 \mathrm{kDa} 11 \mathrm{~S}$ globulin (Act d 12) are the major allergens in green kiwifruit. In gold kiwifruit, kiwellin (Act d 5) of 28 $\mathrm{kDa}$ has been identified to be the main allergen (16). Kiwifruit can cause a variety of allergic symptoms ranging from mild oral allergy syndrome to serious life-threatening anaphylaxis (17). Kiwifruit allergy may be complicated by birch pollinosis and latex allergy (18). Kiwifruit may also be cross-reactive with other foods such as avocado (19), banana (20), and peanut (21). Thus, kiwifruit can cause various allergic symptoms and is known to contain many types of allergens. The diverse symptoms might develop because of the involvement of diverse sensitization pathways. However, the association between these individual allergen components and sensitization pathways, especially percutaneous sensitization, is unclear.

Therefore, in this study, we aimed to identify kiwifruit proteins that percutaneously sensitize mice, for which we applied crude kiwifruit extract epidermally to the dorsal skin of mice and used serum specific immunoglobulin E (IgE) and immunoglobulin $\mathrm{G} 1$ (IgG1) as sensitization markers.

\section{Materials and methods}

\section{Materials}

Enzyme-linked immunosorbent assay (ELISA) plates were purchased from AGC (Tokyo, Japan). Horseradish peroxidase (HRP)-conjugated goat anti-mouse $\operatorname{IgE}$ antibody was purchased from Southern Biotech (Birmingham,AL, USA).HRP-conjugatedgoatanti-mouse IgG1 antibody was purchased from Bethyl Laboratories (Montgomery, TX, USA). 3,3,5,5-Tetramethylbenzidine (TMB) peroxidase substrate was purchased from Kirkegaard \& Perry Laboratories (KPL; Gaithersburg, $\mathrm{MD}$, USA). Coomassie brilliant blue (CBB) R-250, Bradford protein assay reagent, and pentobarbital sodium salt were purchased from Nacalai Tesque (Kyoto, Japan). Enhanced chemiluminescence (ECL) western blotting substrates and X-ray films (Amersham Hyperfilm MP) were purchased from GE Healthcare (Chalfont St. Giles, UK). Luminata Crescendo Western HRP Substrate was purchased from Merck Millipore (Burlington, MA, USA). Immunoreactive enhancers, Can Get Signal solutions 1 and 2, were purchased from TOYOBO (Osaka, Japan). All other chemicals used in this study were of the highest purity available.

\section{Preparation of kiwifruit extract and determination of protein content}

Green and gold kiwifruits were purchased from a supermarket near the university. They were homogenized using a commercial food mixer and extracted through four layers of gauze. The protein content of the obtained extracts was determined using the Bradford method (22) using protein assay CBB solution (Nacalai Tesque, Kyoto, Japan), and the absorbance was measured at $595 \mathrm{~nm}$.

\section{Animal studies}

Six-week-old female BALB/c mice were purchased from Japan SLC Inc. (Shizuoka, Japan) and used for percutaneous sensitization treatment, as shown in Fig. 2. The mice were fed a commercial chow without kiwifruit proteins (MF, Oriental Yeast, Tokyo, Japan) and water was provided ad libitum. The mice were acclimated for 7 days before the start of the experiment. All animal experiments were approved by the Kindai University Animal Experiment Commission (Approval No. KAAG-26-004).

\section{Percutaneous sensitization}

After shaving the occipital region of the mice with an electric shaver under anesthetic conditions, the remaining hair was removed using a hair removal cream (Veet, 
Reckitt Benckiser, Berkshire, UK), and tape stripping was performed 10 times. A mixture of midazolam (Astellas Pharma, Tokyo, Japan), butorphanol (Meiji Pharmaceutical, Tokyo, Japan), and medetomidine (Nippon Zenyaku Kogyo, Fukushima, Japan) was used for giving anesthesia. The treatment was performed weekly. Transdermal sensitization was induced by applying respective samples to the skin after treatment. Three groups such as control, green kiwifruit, and gold kiwifruit were established ( $n=5$ or 6 ).

The control group received 5\% sodium dodecyl sulfate (SDS) alone, the green kiwifruit group received $4 \mathrm{mg} / \mathrm{mL}$ of green kiwifruit extract in 5\% SDS, and the gold kiwifruit group received $4 \mathrm{mg} / \mathrm{mL}$ of gold kiwifruit extract in $5 \%$ SDS. Four times a week for a total of 5 weeks, $50 \mu \mathrm{L}$ of the sample was applied to the epidermis using a micropipette. Then, the serum was collected weekly. At 5 weeks, all the mice were anesthetized by injecting sodium pentobarbital salt intraperitoneally. The mice were then sacrificed by cervical dislocation. The mice were weighed weekly to determine whether their growth was inhibited.

\section{Enzyme-linked immunosorbent assay}

$\mathrm{IgE}$ and $\mathrm{IgG1}$ levels in the sera of mice were measured by performing ELISA. Green and gold kiwifruit extracts (20 $\mu \mathrm{g} / \mathrm{mL}$ each) in phosphate-buffered saline (PBS) were added to the ELISA plate (AGC: Tokyo, Japan) and solidified overnight at $4^{\circ} \mathrm{C}$. The plates were blocked with $1 \%$ BSA in $0.1 \%$ Tween 20 (PBST) for $1 \mathrm{~h}$ at room temperature and washed three times with $100 \mu \mathrm{L}$ of PBST; $50 \mu \mathrm{L}$ of each of the sera diluted with $1 \%$ BSA was added and incubated at $37^{\circ} \mathrm{C}$ for $1 \mathrm{~h}$. After washing five times with PBST, $50 \mu \mathrm{L}$ of secondary antibodies were added respectively, and incubated at $37^{\circ} \mathrm{C}$ for $1 \mathrm{~h}$. After washing five times with PBST, the bound secondary antibody was detected by reacting with $50 \mu \mathrm{L}$ of TMB-peroxidase substrate (KPL, Gaithersburg, MD, USA). The response was stopped by adding $50 \mu \mathrm{L}$ of $1 \mathrm{M}$ phosphate, and the signal was amplified. Sera were diluted 100 -fold for $\mathrm{IgE}$ measurements and 5,000-fold for measuring $\mathrm{IgG} 1$ levels. For secondary antibodies, 8,000-fold diluted goat anti-mouse HRP-conjugated IgE (Southern Biotech: Birmingham, USA) and $1 \%$ BSA were used to measure $\operatorname{IgE}$ levels, whereas 50,000-fold diluted goat anti-mouse IgG1 HRP-conjugated antibody (Bethyl Laboratories, Montgomery, TX, USA) and $1 \%$ BSA were used to measure IgG1 levels. The absorbance of each well was measured at $450 \mathrm{~nm}$ using a plate reader (Wallac ARVO SX 1420 multilabel counter, PerkinElmer, Waltham, MA, USA). The absorbance of two wells was measured for each reading, and their means were used for statistical analysis.

\section{Electrophoresis and immunoblotting}

Green kiwifruit and gold kiwifruit extracts were separated by sodium dodecyl sulfate-polyacrylamide gel electrophoresis
(SDS-PAGE) (23) by running at $200 \mathrm{~V}$ (constant voltage) for approximately $35 \mathrm{~min}$. For the confirmation of protein patterns, SDS-PAGE gels were stained with CBB. After SDS-PAGE, the gels were transferred to polyvinylidene difluoride (PVDF) membranes for immunoblotting (Immobilon-P; Millipore, Billerica, MA, USA) in a semi-dry manner at $15 \mathrm{~V}$ (constant voltage) for $30 \mathrm{~min}$ (24).

Blocking was performed by immersing the PVDF membrane in 5\% skim milk solutions dissolved in PBST for $1 \mathrm{~h}$. For IgE measurement, the membrane was washed two times with PBST for $5 \mathrm{~min}$. The serum diluted 100 -fold using Can Get Signal2 (Toyobo: Osaka, Japan) and the serum diluted 200-fold using skim milk solution were reacted overnight at $4^{\circ} \mathrm{C}$ for IgG1 measurement. After three 10-min washes with PBST, IgE was measured using an HRP-conjugated rat monoclonal [23G3] anti-mouse $\mathrm{IgE}$ antibody (Abcam: Cambridge, UK) that was diluted 4,000-fold by Can Get Signal2 and reacted with the protein surface of the PVDF membrane for $1 \mathrm{~h}$. IgG1 was measured using an HRP-conjugated goat anti-mouse $\operatorname{IgG1}$ antibody diluted 50,000fold with skim milk solution. Four 10-min washes with PBST were followed by reaction with Luminata Crescendo Western HRP Substrate (Millipore Corporation: Billerica MA01821, USA for IgE determination) and Amersham ECL Western Blotting Detection Reagents (GE Healthcare: Chalfont St for IgG1 determination. Giles, UKs) for $1 \mathrm{~min}$, and luminescence signals were detected using X-ray films (Amersham Hyperfilm MP; GE Healthcare).

In addition, the presence of actinidin was confirmed by immunoblotting. After performing SDS-PAGE, blotting, and blocking as described previously, an anti-actinidin antibody diluted five-fold with skim milk was reacted for $1 \mathrm{~h}$. After washing four times for $10 \mathrm{~min}$ with PBST and with Amersham ECL Western Blotting Detection Reagents (GE Healthcare) for $1 \mathrm{~min}$, the emission signal was detected using X-ray films (Amersham Hyperfilm MP; GE Healthcare). The actinidin antibody used was obtained from the allergen eye ELISA II kiwifruit kit (Prima Meat Packers, Ltd., Ibaraki, Japan).

\section{Purification and identification of kiwifruit antigens}

IgG1 binding proteins were purified by ammonium sulfate precipitation, followed by ion-exchange and gel-filtration chromatography to identify percutaneously sensitizing antigens in green kiwifruit. In every purification step, SDS-PAGE and immunoblotting were performed. IgG1-binding proteins were detected using mixed mouse sera. The sera of the mice used were mixed with equal amounts of serum from six green kiwifruit-applied mice collected at week 4 and used as primary antibodies.

\section{Ammonium sulfate precipitate}

Green kiwifruit extract $(100 \mathrm{~mL})$ in buffer A $(10 \mathrm{mM}$ Tris- $\mathrm{HCl}, \mathrm{pH} 7.5,1 \mathrm{mM}$ EDTA) containing the protease 
inhibitor mix (Nacalai Tesque) was freshly prepared for purification. Ammonium sulfate was added stepwise to the above solution, and precipitate was obtained by centrifugation at each step. Samples precipitated with ammonium sulfate $(0-20 \%, 20-40 \%$, and $40-60 \%)$ were recovered and resolved with $10 \mathrm{~mL}$ of buffer A.

\section{Ion-exchange chromatography}

The samples obtained after ammonium sulfate precipitation were desalted using a PD-10 mini-column (GE Healthcare), and then manually prepared ion-exchange chromatography was conducted using Super Q anion-exchange gels (TOSOH, Tokyo, Japan) (bed volume: $5 \mathrm{~mL}$ ) to separate proteins in a stepwise manner using $\mathrm{NaCl}$.

\section{Gel-filtration chromatography}

The samples obtained by ion-exchange chromatography were concentrated using Amicon Ultra-15 $10 \mathrm{~K}$ filters (Merck Millipore, Burlington, MA, USA) and gel-filtration chromatography (TOSOH G3000SW) connected to high-pressure liquid chromatography (HPLC) (Hitachi L-6200) for further purification (flow rate: $0.5 \mathrm{~mL} / \mathrm{min}$ ). Elution fractions $(0.5 \mathrm{~mL} /$ fraction $)$ from gel-filtration chromatography were used to perform CBB staining and immunoblotting after resolving the proteins by SDSPAGE as described earlier.

\section{$N$-terminal amino acid sequence analysis}

The sample obtained from gel-filtration chromatography was subject to SDS-PAGE and transferred onto a PVDF membrane as described earlier. The PVDF membrane was stained with Ponceau S, and the band corresponding to the IgG1 binding protein was cut and collected. The $\mathrm{N}$-terminal amino acid sequence of the sensitizing antigen was determined using Edman degradation (25) (analyzed at Hokkaido System Science, Sapporo, Japan).

\section{Statistical analysis}

Statistical differences were determined using Tukey's multiple comparison test and Dunnett's test. A $P<0.05$ was considered statistically significant. Statistical analysis was performed using the StatView 5.0 software (SAS Institute, Cary, NC, USA).

\section{Results}

\section{Protein patterns of green kiwifruit and gold kiwifruit}

The protein content in green kiwifruit and gold kiwifruit was confirmed by CBB staining after SDS-PAGE, and the patterns of protein bands between the two species were generally similar, although there were slight differences (Fig. 1). The major protein bands in these two species included 26, 24, 23, 20, and $15 \mathrm{kDa}$ bands.

\section{Detection of changes in kiwifruit-specific $\lg E$ and $\lg G \mid$ antibodies determined using ELISA}

The experimental animal scheme is summarized in Fig. 2. Body weights were measured weekly to ensure that the mice were not under excessive stress by the application of allergens. Thus, body weights gradually increased normally over time. In addition, no statistically significant difference was observed in body weight changes between the groups, and hence, the mice were raised normally and

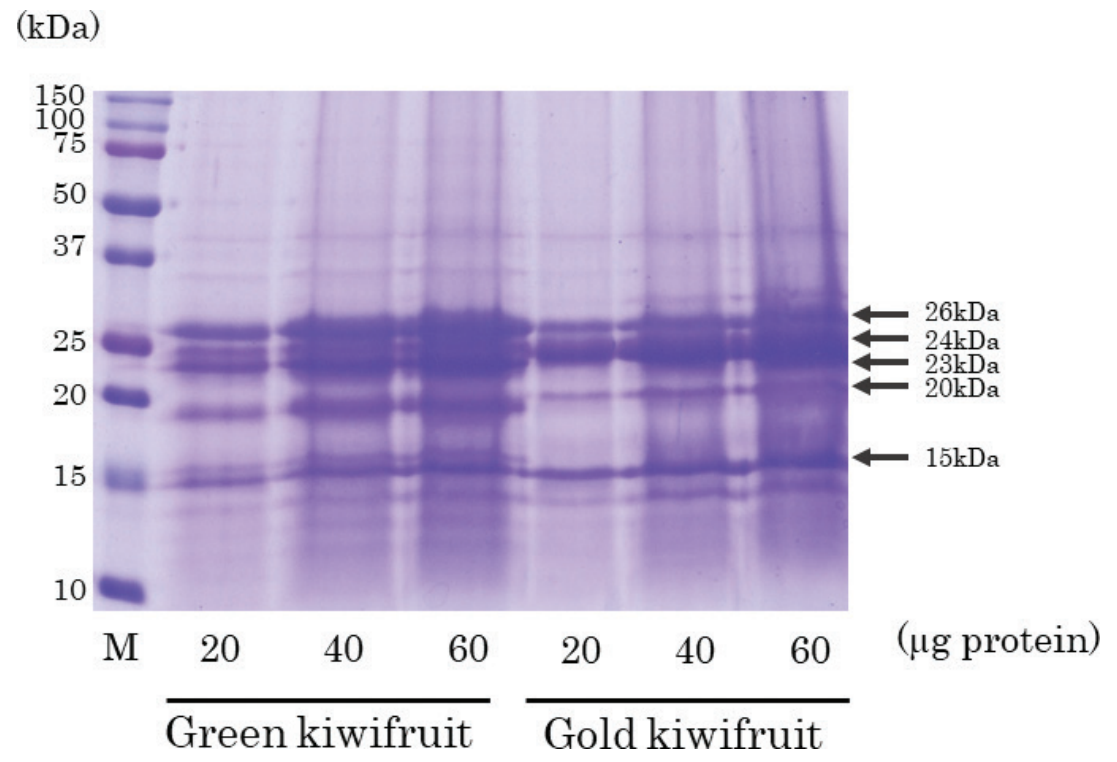

Fig. 1. Coomassie brilliant blue staining of green kiwifruit and gold kiwifruit proteins. Green kiwifruit and gold kiwifruit proteins $(20,40,60 \mu \mathrm{g} / \mathrm{lane})$ were subject to sodium dodecyl sulfate-polyacrylamide gel electrophoresis and stained with Coomassie brilliant blue. Detailed information is given in 'Materials and methods'. 


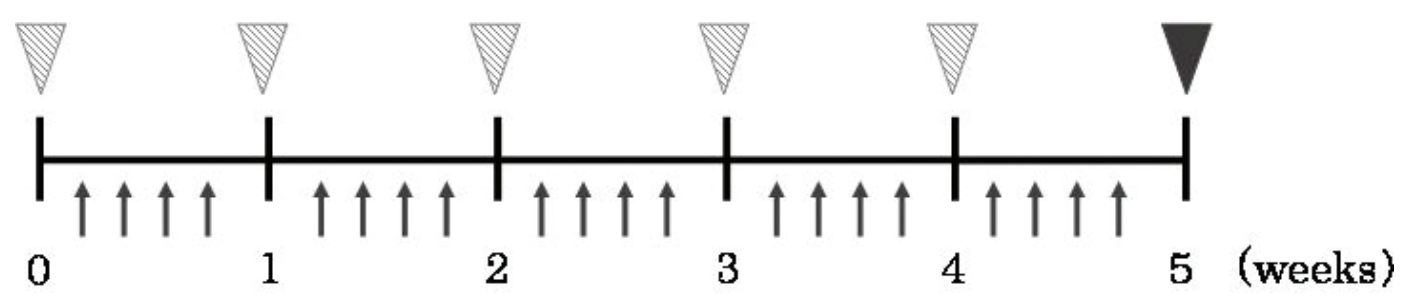

Topical application of the samples (four times a week)

Shaving and Tape Stripping, Collecting the serum (once a week)

Sacrificed (The last day)

\begin{tabular}{|llllll|}
\hline \multicolumn{2}{c}{ Control } & group & 5\%SDS alone & $(\mathrm{n}=5)$ \\
Green & kiwifruit & group & : Green kiwifruit extract in 5\%SDS & $(\mathrm{n}=6)$ \\
Gold & kiwifruit & group & : Gold kiwifruit extract in 5\%SDS & $(\mathrm{n}=5)$ \\
\hline
\end{tabular}

Fig. 2. Schema of the percutaneous sensitization protocol. Detailed information is given in 'Materials and methods'.

(a)

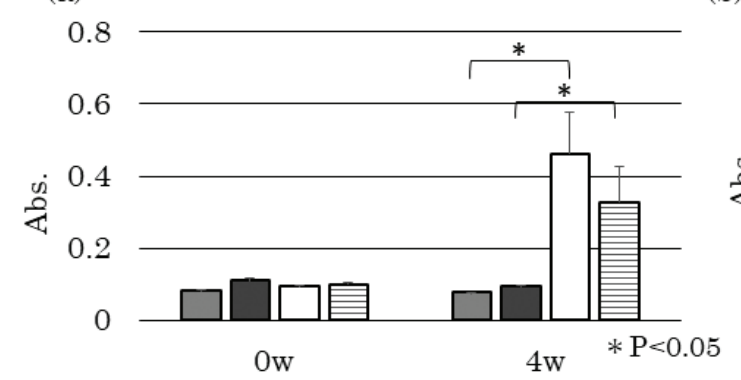

$\square$ Control (Green Kiwifruit) group

$\square$ Control (Gold Kiwifruit) group

$\square$ Green Kiwifruit group

目Gold Kiwifruit group (b)

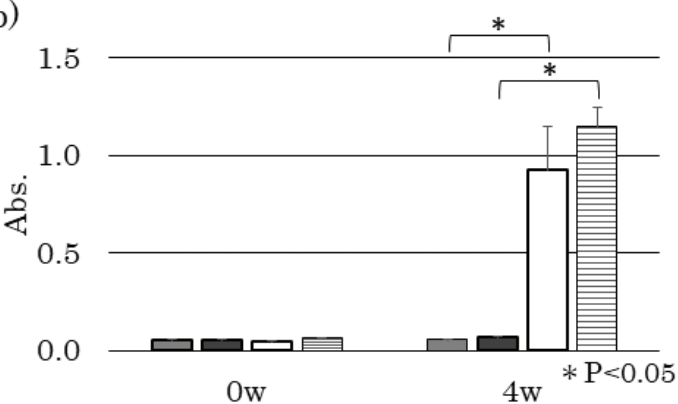

$\square$ Control (Green Kiwifruit) group

$\square$ Control (Gold Kiwifruit) group

$\square$ Green Kiwifruit group

目 Gold Kiwifruit group

Fig. 3. Effect of percutaneous sensitization with kiwifruit extracts on kiwifruit-specific antibody levels in mice sera. Kiwifruit protein-specific IgE levels (a) and IgG1 levels (b) in control and kiwifruit groups at 0 and 4 weeks were determined using ELISA performed with each kiwifruit protein coated plates. Sera from the control group were measured two-way, one by using green kiwifruit as a solid-phase (Control (Green Kiwifruit)) and the other by using gold kiwifruit as a solid-phase (Control (Gold Kiwifruit)). Absorbance data are expressed as means \pm standard deviations; control group $(n=5)$, green kiwifruit group $(n=6)$, gold kiwifruit group $(n=5) . * P<0.05$.

no differences in growth were observed depending on the difference in the applied samples (data not shown).

Sera from weeks 0 and 4 were used to assess antigen-specific $\operatorname{IgE}$ and $\operatorname{IgG} 1$ in sera by performing antigen solid-phase ELISA. Specific IgE (Fig. 3a) and IgG1 (Fig. 3b) levels were significantly higher in both green kiwifruit and gold kiwifruit groups compared with the control group $(P<0.05)$. Sera from the control group were measured in two ways, one by using green kiwifruit as a solid-phase (green control) and the other by using gold kiwifruit as a solid-phase (gold control). No significant difference was observed in these values (Fig. 3a and 3b).

\section{Detection of $\lg E$ - and $\lg G$ I-binding kiwifruit proteins using immunoblotting}

To determine which proteins in kiwifruits are bound by $\mathrm{IgE}$ and $\mathrm{IgG} 1$ antibodies produced in the serum of mice, immunoblots were performed with serum of 4 weeks. Green (Fig. 4A) and gold (Fig. 4B) kiwifruit proteins were run separately and used for immunoblotting. 
A. a)

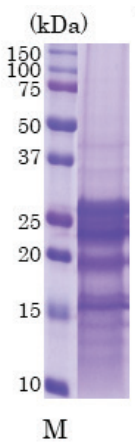

B. a)

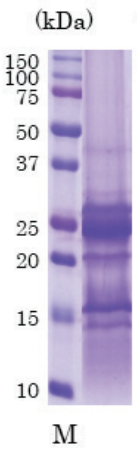

b)

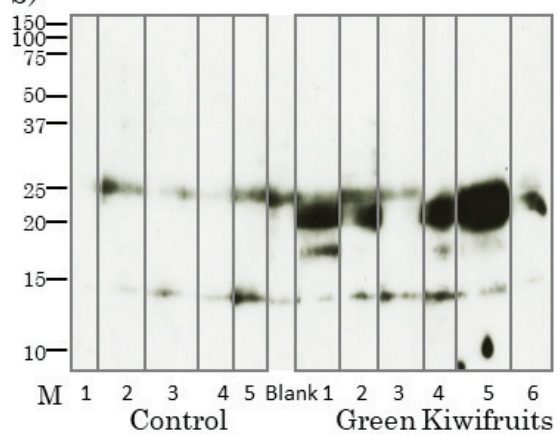

b) groups

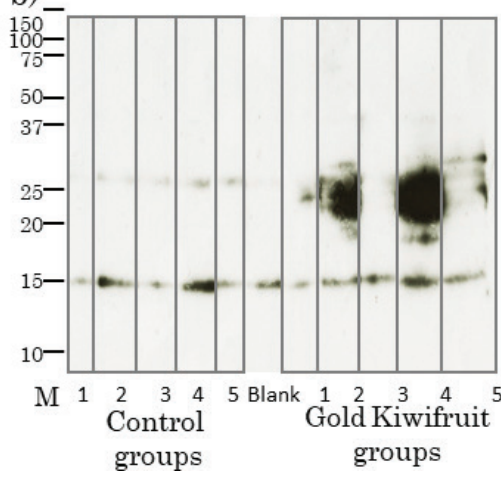

c)

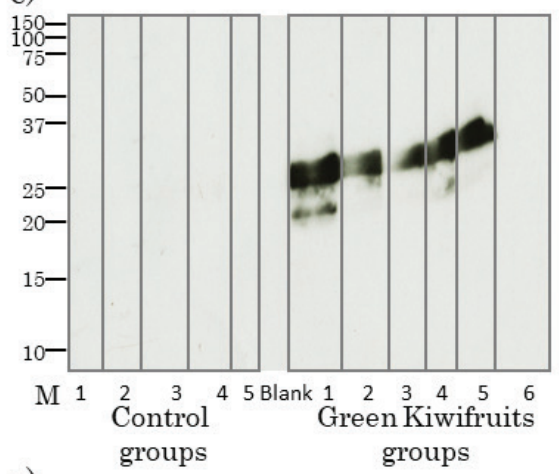

c)

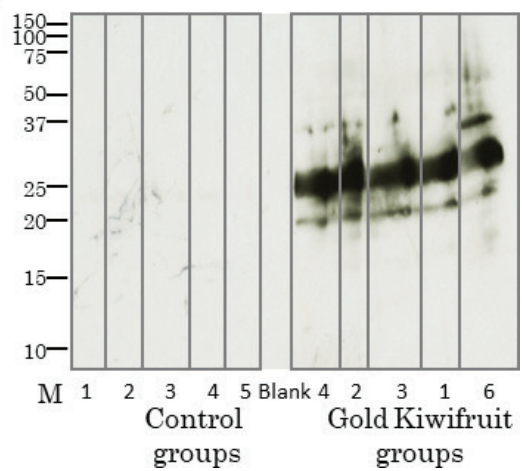

Fig. 4. Detection of $\operatorname{IgE}$ and $\operatorname{IgG1}$, binding to two species of kiwifruit proteins using immunoblotting. (A) Coomassie brilliant blue staining of green kiwifruit proteins (a). The numbers indicate the individual mice in the control and green kiwifruit groups. Immunoblotting using green kiwifruit proteins, individual mice sera and horseradish peroxidase (HRP)-labeled anti-mouse IgE antibody (b). Immunoblotting using green kiwifruit proteins, individual mice sera, and HRP-labeled anti-mouse IgG1 antibody (c). (B) The numbers indicate the individual mice in control and gold kiwifruit groups. Coomassie brilliant blue staining of gold kiwifruit proteins (a). Immunoblotting using gold kiwifruit proteins, individual mice sera, and HRP-labeled anti-mouse IgE antibody (b). Immunoblotting using gold kiwifruit proteins, individual mice sera, and HRP-labeled anti-mouse IgG1 antibody (c).

Immunoblots for green kiwifruit proteins revealed multiple antigen-specific IgE (Fig. 4A(b)) and IgG1 bands in the green kiwifruit group (Fig. 4A(c)). Similarly, immunoblots for gold kiwifruit proteins revealed multiple antigen-specific $\operatorname{IgE}$ (Fig. 4B(b)) and IgG1 bands in the gold kiwifruit group (Fig. 4B(c)). In the case of IgE, bands reactive to $26 \mathrm{kDa}$ and $15 \mathrm{kDa}$ were also detected in sera from the control groups (Fig. 4A(b) and Fig. 4B(b)). These bands appeared to represent non-specific binding proteins, probably the second antibody directly binding to kiwifruit proteins. In addition, a dense band in the region of $20-25 \mathrm{kDa}$ was observed, and was detected in both the kiwifruit groups (Fig. 4A(b) and Fig. 4B(b)). In the case of $\operatorname{IgG} 1$, non-specific binding proteins were not observed, and at least two dense IgG1 bands were detected in the $20-25 \mathrm{kDa}$ region in both the kiwifruit groups (Fig. 4A(c) and Fig. 4B(c)). IgE and IgG1 bands were also detected in the region of $18-20 \mathrm{kDa}$ in sera from several individual mice.

In case of both IgE and IgG1, strong antibody-binding protein(s) in the region of $20-25 \mathrm{kDa}$ were commonly detected, specifically in sera from both the kiwifruit groups, suggesting that both $\operatorname{IgE}$ and $\operatorname{IgG} 1$ bound to the same protein(s). These proteins were considered to be transdermal sensitizing antigen candidates from both the kiwifruits in this mouse model system.

Confirmation of cross-reactions between two species of kiwifruit To confirm the cross-reactions between two species of kiwifruit, we investigated whether $\operatorname{IgE}$ and $\operatorname{IgG} 1$ antibodies in mice sera applied with each kiwifruit cultivar react similarly to the other kiwifruit cultivar proteins. ELISA analysis showed that serum IgE (Fig. 5A(a)) and $\operatorname{IgG1}($ Fig. $5 \mathrm{~A}(\mathrm{~b}))$ from the green kiwifruit group responded almost equally to green and gold kiwifruit proteins. Serum IgE (Fig. 5B(a)) and IgG1 (Fig. 5B(b)) from the gold kiwifruit group also reacted almost equally to green and gold kiwifruit proteins. Similarly, cross-reactive antibody-binding proteins that appeared to be almost identical were found in each cultivar in immunoblot analyses (Fig. 6). Therefore, the antibody produced for green kiwifruit in this model system cross-reacted with the allergen molecules in the gold kiwifruit proteins, and vice versa (Fig. 6). 
A.

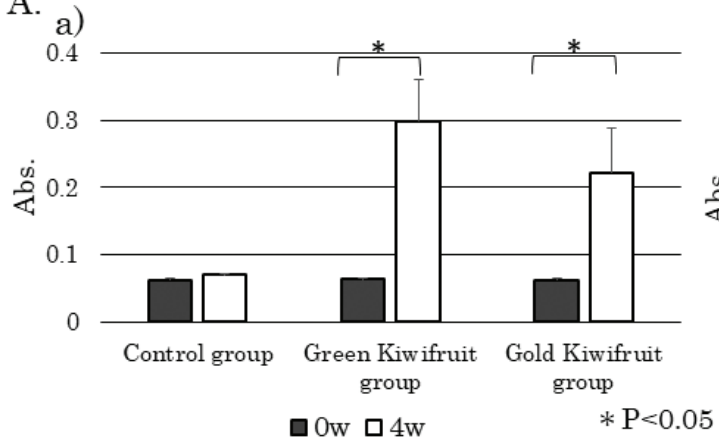

b)

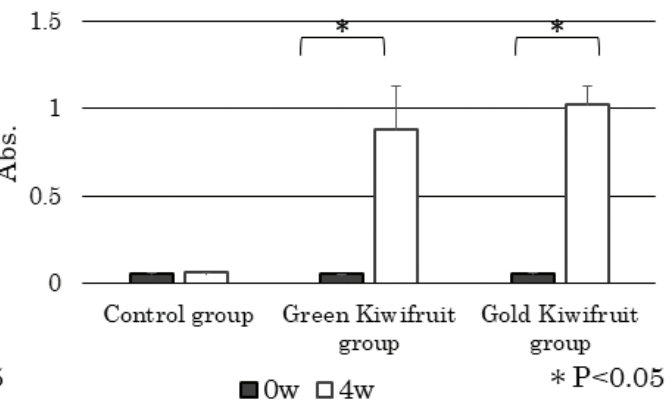

B.

a)

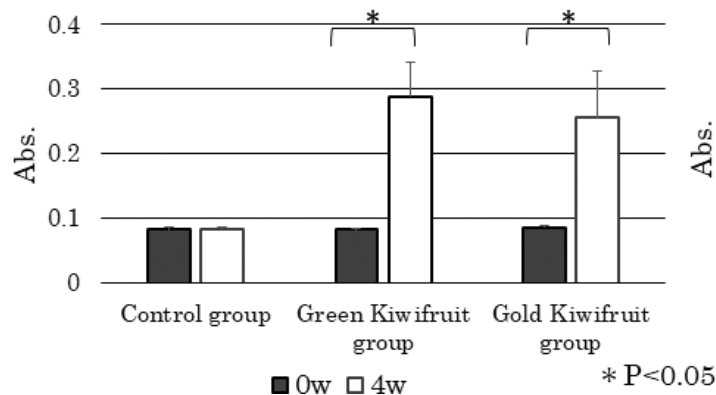

b)

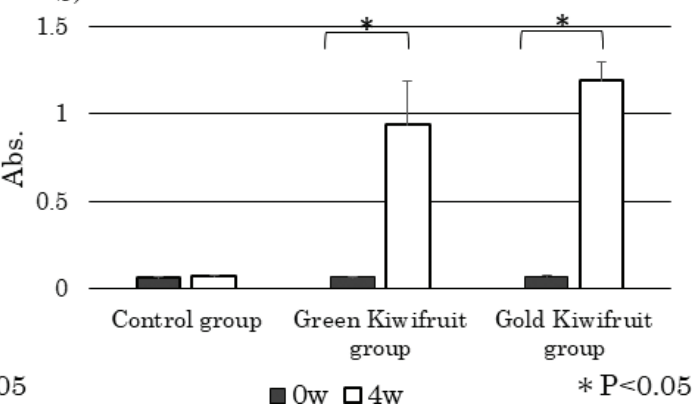

Fig. 5. Confirmation of cross-reactions between two species of kiwifruit. (A) The serum IgE (a) and IgG1 levels (b) in control and kiwifruit groups at 0 and 4 weeks were determined using enzyme-linked immunosorbent assay (ELISA) performed with green kiwifruit protein-coated plates. (B) Serum IgE levels (a) and IgG1 levels (b) in control and kiwifruit groups at 0 and 4 weeks were determined using ELISA performed with gold kiwifruit protein-coated plates. Absorbance data are expressed as means \pm standard deviations; control group $(n=5)$, green kiwifruit group $(n=6)$, gold kiwifruit group $(n=5)$. ${ }^{*} P<0.05$.

\section{Purification of $\lg G \mid$-binding proteins from green kiwifruit}

As the kiwifruit protein bands (approximately $20-25 \mathrm{kDa}$ ) that bind $\operatorname{IgE}$ and $\operatorname{IgG} 1$ were found to be the same, more sensitive IgG1 was selected for the subsequent purification and identification of sensitizing antigens.

Green kiwifruit extract was fractionated using ammonium sulfate. CBB staining (Fig. 7A(a)) and immunoblotting (Fig. 7A(b)) of each sample performed after ammonium sulfate precipitation revealed that the 20-25 kDa IgG1-binding protein bands were most abundant in the samples precipitated with $0-20 \%$ ammonium sulfate. The sample precipitated with $0-20 \%$ ammonium sulfate was collected and desalted, and anion-exchange chromatography was performed.

CBB staining and immunoblotting (Fig. 7B) of the ion-exchange chromatography fractions revealed that the proteins in the region of $20-25 \mathrm{kDa}$ were present in the fraction eluted with the buffer containing $0.05 \mathrm{M}$ and 0.1 $\mathrm{M} \mathrm{NaCl}$. These two fractions were separately used for subsequent gel-filtration chromatography.

The samples showing a single band and subject to ion-exchange chromatography with $0.05 \mathrm{M} \mathrm{NaCl}$ were collected and further subject to gel-filtration HPLC using G3000SW gel-filtration HPLC columns after concentration. A major IgG1-binding protein band with a molecular weight of approximately $23 \mathrm{kDa}$ was identified in fraction numbers 14-19 (Fig. 8A). A minor IgG1-binding protein band of $18 \mathrm{kDa}$ was also detected in fractions numbers 16 and 17 (Fig. 8A).

The samples subject to ion-exchange chromatography with $0.1 \mathrm{M} \mathrm{NaCl}$ were similarly fractionated using gel-filtration columns, and two IgG1 binding protein bands ( 23 and $24 \mathrm{kDa}$ ) were confirmed in the fraction numbers 14-17 (Fig. 8B).

\section{$\mathrm{N}$-terminal amino acid sequence analysis of green kiwifruit- derived $\lg G \mid$ binding proteins $(18,23$, and $24 \mathrm{kDa}$ )}

Fractions eluted from gel-filtration columns were transferred to PVDF membranes, and Edman degradation analysis was performed to identify these protein bands. The N-terminal sequences of $23 \mathrm{kDa}$ and $24 \mathrm{kDa}$ proteins were found to be 'TCSPQPGG', whereas the N-terminal amino acid sequence of the protein of $18 \mathrm{kDa}$ molecular weight was 'ISSCNGPP'. A homology search using BLAST for proteins with matched $\mathrm{N}$-terminal sequences was performed, and all the three samples matched with the N-terminal proximal sequence of kiwellin (Act d 5), a cysteine-rich cell wall-related protein (Fig. 9). 
A.

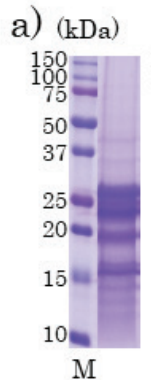

B.

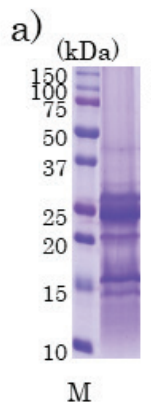

b)

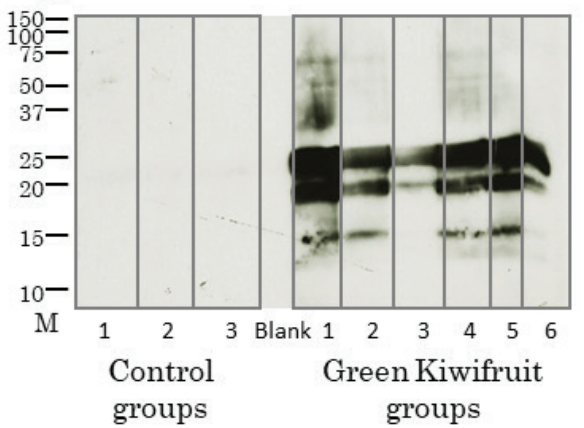

b)

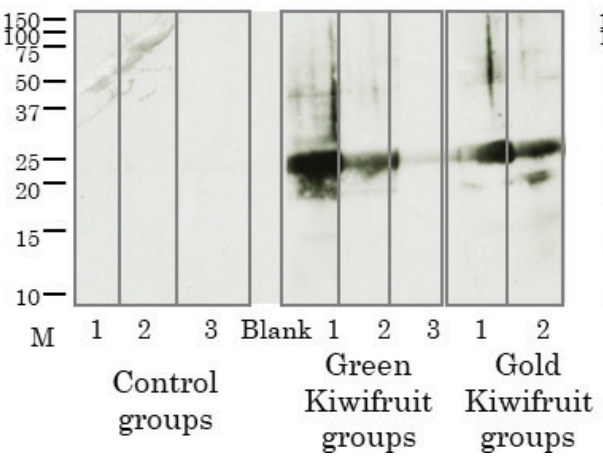

c)

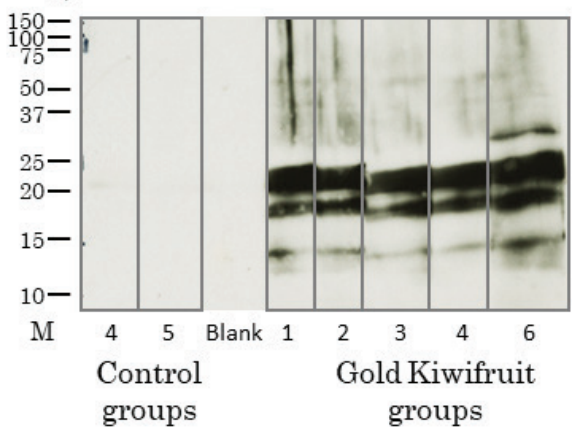

c)

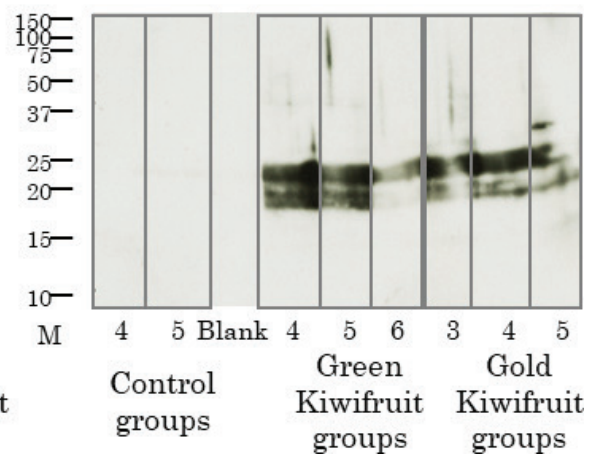

Fig. 6. Confirmation of cross-reactions between two species of kiwifruit using immunoblotting. (A) Coomassie brilliant blue staining of green kiwifruit proteins (a). The numbers indicate the individual mice in control and each of kiwifruit groups. Immunoblotting using green kiwifruit proteins, individual mice sera, and horseradish peroxidase (HRP)-labeled anti-mouse IgG1 antibody (b, c). (B) The numbers indicate the individual mice in control and each of kiwifruit groups. Coomassie brilliant blue staining of gold kiwifruit proteins (a). Immunoblotting using gold kiwifruit proteins, individual mice sera, and HRP-labeled anti-mouse IgG1 antibody (b, c).

\section{Discussion}

Kiwifruit is a popular fruit of the genus Actinidia that contains abundant bioactive substances such as dietary fiber, carbohydrates, natural sugars, vitamins, minerals, and antioxidants, and hence, globally consumed (11). However, many food allergy cases have also been reported to be caused by kiwifruit (13). Whether kiwifruit proteins can transdermally sensitize a mouse model has not been fully investigated. Therefore, in this study, we demonstrated transdermal sensitization to kiwifruit proteins in a mouse model.

ELISA showed significantly high levels of both kiwifruit-specific IgE and $\operatorname{IgG} 1$ antibodies in both the kiwifruit groups. Immunoblotting revealed several major IgE- and IgG1-binding protein bands of around 18 and 20-25 kDa. Cross-reactivity was observed between the two varieties of kiwifruits. Therefore, the protein bands detected could be transdermal sensitization antigens found in kiwifruits. In addition, no significant difference was found between the $\operatorname{IgE}$ and $\operatorname{IgG} 1$ levels produced between green and gold kiwifruits, and therefore, no difference in the transdermal sensitization ability between these varieties of kiwifruits was confirmed.
Using green kiwifruit-specific $\operatorname{IgG} 1$ binding as an indicator, three transdermal sensitizing allergens of 18, 23, and $24 \mathrm{kDa}$ molecular weights were purified and identified, and their partial amino acid sequence was found to be similar to that of kiwellin. Among them, the $18-\mathrm{kDa}$ sample was considered to be a degradation product of kiwellin called KiTH (26). The purified and identified transdermal sensitizing allergens of 23 and $24 \mathrm{kDa}$ were identical to the $\mathrm{N}$-terminal amino acid sequence of kiwellin. The differences between the two molecules might be because of post-translational modifications or limited proteolysis around the $\mathrm{C}$-terminal region of the proteins; however, the details of the molecular weight differences between the two allergens are unknown.

In the past three decades, the incidence of kiwifruit allergy due to actinidin, kiwellin and thaumatin-like protein has increased (27). The function of kiwellin, a putative transdermal sensitizing allergen, is unclear; however, this cysteine-rich protein is one of the major proteins present in large quantities in the edible parts of the kiwifruit (28). Kiwellin acts as a cell wall or a plant defense protein to offset foreign effector activities with high specificity (29). A recent study using transcriptomic 
A.

a)

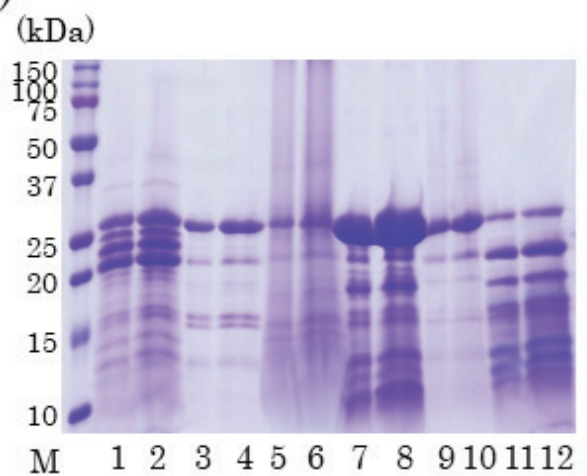

b)

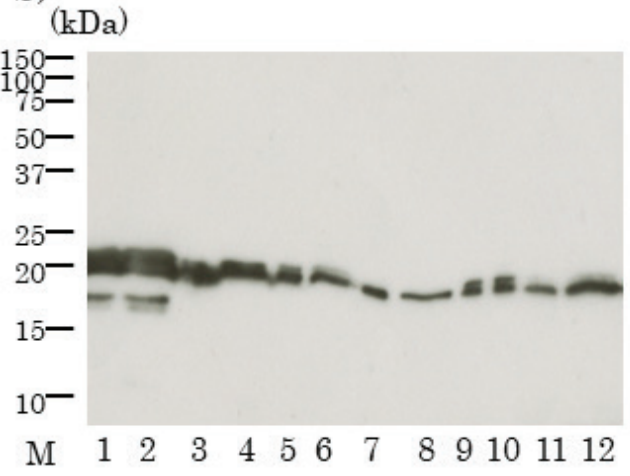

B.

a)

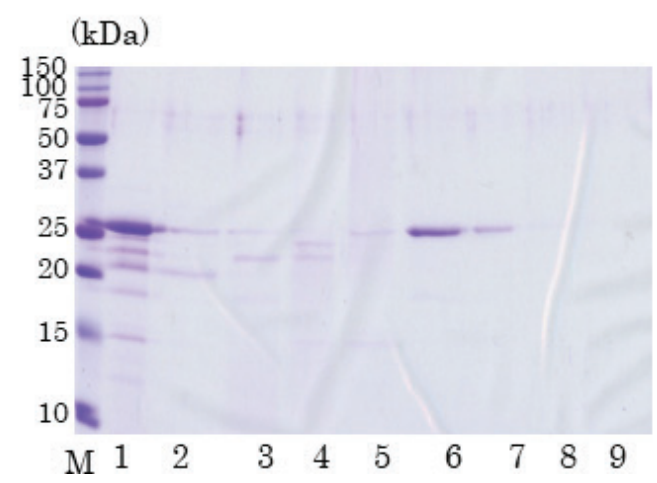

b)

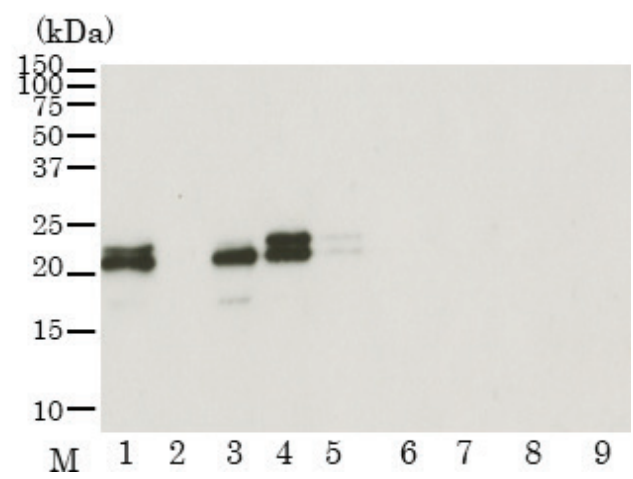

Fig. 7. The fraction of kiwifruit proteins using ammonium sulfate precipitation and ion-exchange chromatography. Coomassie brilliant blue staining of fractionated kiwifruit proteins $(A(a), B(a))$. Immunoblotting using fractionated kiwifruit proteins, mixed mice sera, and horseradish peroxidase-labeled anti-mouse $\operatorname{IgG1}$ antibody (A(b), B(b)). (A) Lanes 1, 3, 5, 7, 9, and 11 were administered $5 \mu \mathrm{L}$ of the sample. Lanes $2,4,6,8,10$, and 12 were administered $10 \mu \mathrm{L}$ of the sample. Lane 1 2: the crude extracted sample; Lane 3 4: fractionated samples with 0 20\%; Lanes 7 8: fractionated samples with 20 40\%; Lanes 11 12: fractionated samples with 40 60\% of ammonium sulfate precipitations. Lane 5 6: precipitations of fractionated samples with 0 20\% of ammonium sulfate; Lane 9 10: precipitations of fractionated samples with $20 \sim 40 \%$ of ammonium sulfate precipitations. (B) Lane 1: fractionated sample with 0 20\% ammonium sulfate precipitation; lane 2: flow-through fraction from ion-exchange chromatography; lanes 3 9: sample eluted with buffer A containing 0.05 M (3), 0.1 M (4), 0.2 M (5), 0.3 M (6), 0.4 M (7), 0.5 M (8), and 1.0 M (9) of $\mathrm{NaCl}$.

data showed that the level of kiwellin homolog was strongly increased in potatoes infected with the oomycete of the potato pest fungus (30). Increased expression of the gene encoding kiwellin has also been observed in adult whitefly infected chrysanthemum. Based on this evidence, kiwellin may be a multifaceted broad scaffold protein that counteracts secreted pathogen effectors (29). Interestingly, many plant allergens are known to be biodefense-related proteins belonging to the pathogenesis-related protein family. Kiwellin, which we found as a transdermal sensitizing antigen, may also be categorized as a class of biodefense-related proteins based on their properties. Why such biodefense-related proteins are prone to be allergenic is unknown but plant-animal interactions might be a major factor involved in it.
Kiwellin is a protein consisting of two domains, an $\mathrm{N}$-terminal kissper (residues 1-39) that consists of six cysteines and an N-terminal $4 \mathrm{kDa}$ peptide moiety, and a $\mathrm{C}$-terminal domain that consists of eight cysteines (residues 40-189) (28). Some of the sera specific for kiwellin from green kiwi were more responsive to KiTH than to kiwellin. On the other hand, some sera responded more highly to kiwellin than to KiTH. Thus, kiwellin might have a hidden IgE-binding epitope that becomes available in KiTH, following the removal of kissper, and kissper might be an IgE-binding epitope by itself (26). In ripe green kiwifruit, kiwellin is degraded by the cysteine protease actinidin, generating a 4-kDa N-terminal domain kispper and a $16-\mathrm{kDa}$ C-terminal domain KiTH (31). Actinidine is also abundant in kiwifruit and accounts for more than $50 \%$ of the mature 
A.

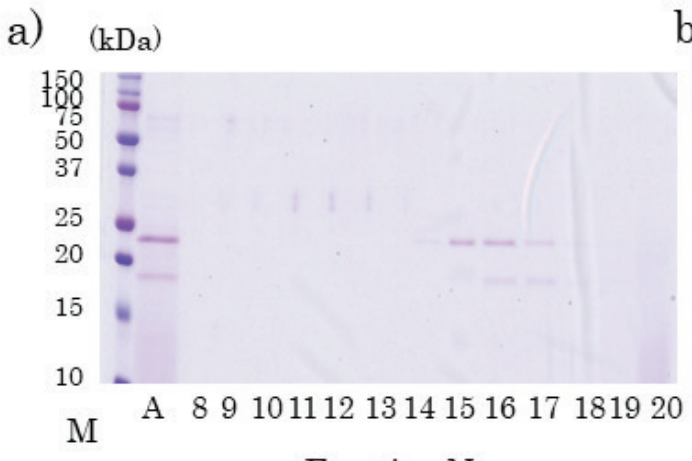

b) $(\mathrm{kDa})$

Fraction No.

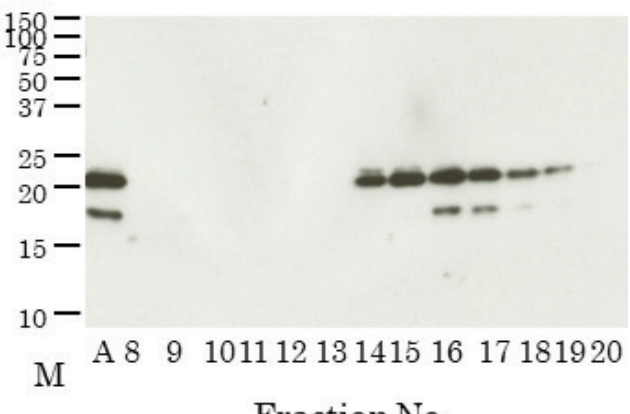

B.

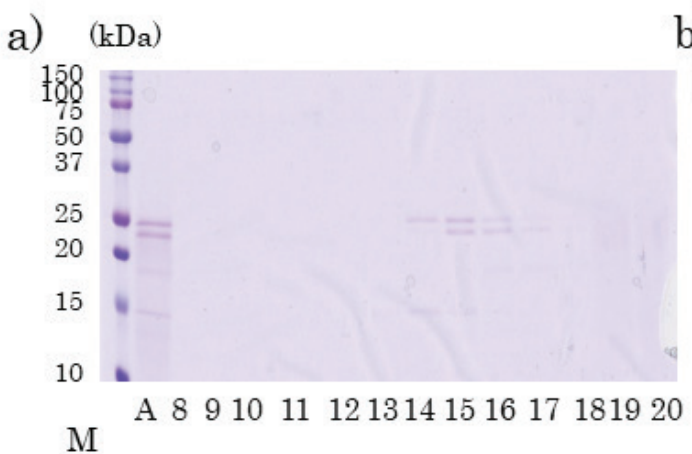

Fraction No. b) $(\mathrm{kDa})$

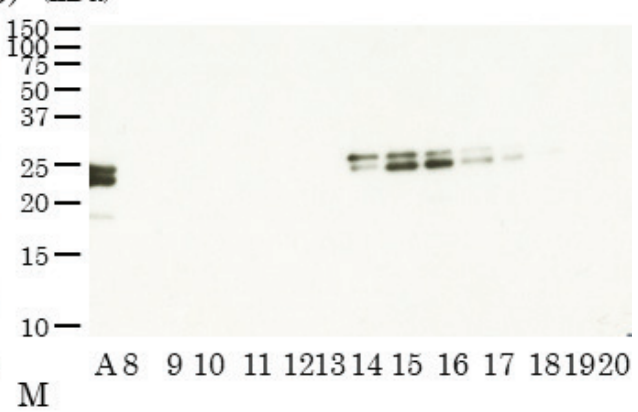

Fraction No.

Fig. 8. Separation of kiwifruit proteins using gel-filtration high-pressure liquid chromatography (HPLC). Coomassie brilliant blue staining of separated kiwifruit proteins (A(a), B(a)). Immunoblotting using fractionated kiwifruit proteins, mice sera, and horseradish peroxidase-labeled anti-mouse IgG1 antibody (A(b), B(b)). (A) Ion-exchanged $0.05 \mathrm{M} \mathrm{NaCl}$ eluted samples with 0-20\% ammonium sulfate precipitation, were collected and fractionated on gel-filtration HPLC columns after concentration. Lane A: concentrated sample; Lanes 8 20: eluted fraction number, respectively. (B) Ion-exchanged $0.1 \mathrm{M} \mathrm{NaCl}$ eluted samples with $0-20 \%$ ammonium sulfate precipitation were collected and fractionated on gel-filtration HPLC columns after concentration. Lane A: concentrated sample; Lanes 8 20: eluted fraction number, respectively.

\section{1 maqlsIIvls IfItlislpp pgasisscng pcrdlndcdg qliciegken ddpevgthic 61 rgttpspqpg gckpsgtltc rgkshptydc sppvtsstpa kltnndfseg gdgggpsecd 121 esyhsnneri valstgwyng gsrcgkmiri tasngksvsa kvvdecdsrh gcdkehagqp 181 pcrnnivdgs navwsalgld knvgvvditw sma}

Fig. 9. Amino acid sequences of Kiwellin. The N-terminal amino acid sequences identified via Edman degradation are shown in red (GeneBank Protein accession no. AGC39167).

kiwifruit protein (32). Because of these properties, actinidine is known as the major allergen in kiwifruit as act $\mathrm{d} 1$ (33). However, in the present study, kiwellin caused IgE and IgG1 production more clearly than actinidin. Therefore, kiwellin might be more sensitizing than actinidin as a transdermal sensitizing antigen in kiwifruit.

\section{Conclusions}

In this study, we showed that both green and gold kiwifruit have transdermal sensitization potential in a murine model system, and identified kiwellin as a new biodefense protein as a sensitization antigen. This molecule is already known as a minor allergen of kiwifruit for humans but could be a major allergen in transdermal sensitization. These findings provide new targets for strategies to reduce the risk of allergy caused by kiwifruit.

\section{Acknowledgments}

The auhors thank Editage (www.editage.jp) for English language editing. 


\section{Conflict of interests and funding}

All authors declare that they have no competing interests. The authors have not received any funding or benefits from industry to conduct this study.

\section{Funding}

This work was supported by JSPS KAKENHI (Grantin-Aid for Scientific Research (C)) Grant Numbers JP25450187 and JP16K07756 to T. M. This study was also supported in part by a grant from the Agricultural Technology and Innovation Research Institute (ATIRI), Kindai University.

\section{References}

1. Sicherer SH, Sampson HA. Food allergy: a review and update on epidemiology, pathogenesis, diagnosis, prevention, and management. J Allergy Clin Immunol 2018; 141: 41-58. doi: 10.1016/j.jaci.2017.11.00

2. Pabst O, Mowat MA. Oral tolerance to food protein. Mucosal Immunol 2012; 5: 232-9. doi: 10.1038/mi.2012.4

3. Galli SJ, Tsai M. IgE and mast cells in allergic disease. Nat Med 2012; 18: 693-704. doi: 10.1038/nm.2755

4. Matsumoto K, Saito H. Epicutaneous immunity and onset of allergic diseases - per-'eczema'tous sensitization drives the allergy march. Allergol Int 2013; 62: 291-6. doi: 10.2332/ allergolint.13-RAI-0603

5. Chinuki Y, Morita E. Wheat-dependent exercise-induced anaphylaxis sensitized with hydrolysed wheat protein in a soap. Allergol Int 2012; 61: 529-37. doi: 10.2332/allergolint.12-RAI-0494

6. Yagami, A, Suzuki K, Nakamura M, Sano A, Iwata Y, Kobayashi $\mathrm{T}$, et al. Case of anaphylactic reaction to soy following percutaneous sensitization by soy-based ingredients in cosmetic products. J Dermatol 2015; 42: 917-18. doi: 10.1111/1346-8138.12966

7. Inomata N, Nagashima M, Hakuta A, Aihara M. Food allergy preceded by contact urticaria due to the same food: involvement of epicutaneous sensitization in food allergy. Allergol Int 2015; 64: 73-8. doi: 10.1016/j.alit.2014.08.005

8. Noti M, Kim BS, Siracusa MC, Rak GD, Kubo M, Moghaddam AE, et al. Exposure to food allergens through inflamed skin promotes intestinal food allergy through the thymic stromal lymphopoietin-basophil axis. J Allergy Clin Immunol 2014; 133: 1390-9. doi: 10.1016/j.jaci.2014.01.021

9. Murakami H, Ogawa T, Takafuta A, Yano E, Zaima N, Moriyama T. Identification of the $7 \mathrm{~S}$ and $11 \mathrm{~S}$ globulins as percutaneously sensitizing soybean allergens as demonstrated through epidermal application of crude soybean extract. Biosci Biotechnol Biochem 2018; 82: 1408-16. doi: 10.1080/09168451.2018.1460573

10. Murakami H, Ogawa T, Takafuta A, Yano E, Zaima N, Moriyama T. Percutaneous sensitization to soybean proteins is attenuated by oral tolerance. J Nutr Sci Vitaminol 2018; 64: 683-6. doi: $10.3177 /$ jnsv. 64.483

11. Shu Q, De Silva UM, Chen S, Peng W, Ahmed M, Lu G, et al. Kiwifruit extract enhances markers of innate and acquired immunity in a murine model. Food Agr Immunol 2008; 19(2): 149-61. doi: 10.1080/09540100802117198

12. Vissers MC, Carr AC, Pullar JM, Bozonet SM. Chapter seventhe bioavailability of vitamin $\mathrm{C}$ from kiwifruit. Adv Food Nutr Res 2013; 68: 125-47. doi: 10.1016/B978-0-12-394294-4.00007-9
13. Chen L, Lucas JS, Hourihane JO, Lindemann J, Taylor SL, Goodman RE. Evaluation of IgE binding to proteins of hardy (Actinidia arguta), gold (Actinidia chinensis) and green (Actinidia deliciosa) kiwifruits and processed hardy kiwifruit concentrate, using sera of individuals with food allergies to green kiwifruit. Food Chem Toxicol 2006; 44(7): 1100-7. doi: 10.1016 / j.fct.2006.01.005

14. Bublin M, Mari A, Ebner C, Knulst A, Scheiner O, Hoffmann-Sommergruber $\mathrm{O}$, et al. IgE sensitization profiles toward green and gold kiwifruits differ among patients allergic to kiwifruit from 3 European countries. J Allergy Clin Immunol 2004; 114(5): 169-75. doi: 10.1016/j.jaci.2004.07.016

15. Fine AJ. Hypersensitivity reaction to kiwifruit (Chinese gooseberry, Actinidia chinensis). Allergy Clin Immunol 1981; 68: 235 7. doi: 10.1016/0091-6749(81)90189-5

16. Wang J, Vanga SK, McCusker C, Raghavan AV. Comprehensive review on kiwifruit allergy: pathogenesis, diagnosis, management, and potential modification of allergens through processing. Compr Rev Food Sci Food Saf 2019; 18(2): 500-13. doi: 10.1111/1541-4337.12426

17. Kerzl R, Simonowa A, Ring J, Ollert M, Mempel M. Life-threatening anaphylaxis to kiwi fruit: protective sublingual allergen immunotherapy effect persists even after discontinuation. J Allergy Clin Immunol 2007; 119(2): 507-8. doi: 10.1016/j. jaci.2006.09.041

18. Bublin M, Pfister M, Radauer C, Oberhuber C, Bulley S, Dewitt AM, et al. Component-resolved diagnosis of kiwifruit allergy with purified natural and recombinant kiwifruit allergens. J Allergy Clin Immunol 2010; 125(3): 687-94. doi: 10.1016/j. jaci.2009.10.017

19. Lee JM, Jeon SA, Lee SY. Clinical characteristics and diagnostic value of specific immunoglobulin $\mathrm{E}$ antibodies in children with kiwi fruit allergy. Allergy Asthma Respir Dis 2013; 1(1): 73-8. doi: 10.4168/aard.2013.1.1.73

20. Sakai Y, Ishihata K, Nakano S, Yamada T, Yano T, Uchida K, et al. Specific detection of banana residue in processed foods using polymerase chain reaction. J Agr Food Chem 2010; 58(14): 8145-51. doi: 10.1021/jf100675c

21. Sirvent S, Cantó B, Gómez F, Blanca N, Cuesta- Herranz J, Canto G, et al. Detailed characterization of act d 12 and act d 13 from kiwi seeds: implication in IgE cross-reactivity with peanut and tree nuts. Allergy 2014; 69(11): 1481-8. doi: 10.1111/ all.12486

22. Bradford MM. A rapid and sensitive method for the quantitation of microgram quantities of protein utilizing the principle of protein-dye binding. J Anal Biochem 1976; 72: 248-54. doi: 10.1006/abio.1976.9999

23. Laemmli UK. Cleavage of structural proteins during the assembly of the head of bacteriophage T4. Nature 1970; 227: 680-5. doi: $10.1038 / 227680 \mathrm{a} 0$

24. Kyhse-Andersen J. Electroblotting of multiple gels: a simple apparatus without buffer tank for rapid transfer of proteins from polyacrylamide to nitrocellulose. J Biochem Biophys Methods 1984; 10: 203-9. doi: 10.1016/0165-022x(84)90040-x

25. Edman P, Hogfeldt E, Sillen GL, Kinell P.-O. Method for determination of the amino acid sequence in peptides. Acta Chem Scand 1950; 4:283-93. doi: 10.3891/acta.chem.scand.04-0283

26. Tuppo L, Giangrieco I, Palazzo P, Bernardi ML, Scala E, Carratore $\mathrm{V}$, et al. Kiwellin, a modular protein from green and gold kiwi fruits: evidence of in vivo and in vitro processing and IgE binding. J Agric Food Chem 2008; 56(10): 3812-17. doi: $10.1021 /$ jf $703620 \mathrm{~m}$ 
27. Maddumage R, Nieuwenhuizen NJ, Bulley SM, Cooney JM, Green SA, Atkinson RG. Diversity and relative levels of actinidin, kiwellin, and thaumatin-like allergens in 15 varieties of kiwifruit (Actinidia). J Agr Food Chem 2013; 61(3): 728-39. doi: 10.1021/ jf304289f

28. Hamiaux C, Maddumage R, Middleditch MJ, Prakash R, Brummell DA, Baker EN, et al. Crystal structure of kiwellin, a major cell wall protein from kiwifruit. J Struct Biol 2014; 187(3): 27681. doi: 10.1016/j.jsb.2014.07.005

29. Han X, Altegoer F, Steinchen W, Binnebesel L, Schuhmacher $\mathrm{J}$, Glatter T, et al. Kiwellin disarms the metabolic activity of a secreted fungal virulence facto. Nature 2019; 565: 650-3. doi: 10.1038/s41586-018-0857-9

30. Draffehn AM, Li L, Krezdorn N, Ding J, Lübeck J, Strahwald $\mathrm{J}$, et al. Comparative transcript profiling by SuperSAGE identifies novel candidate genes for controlling potato quantitative resistance to late blight not compromised by late maturity. Front Plant Sci 2013; 4: 423. doi: 10.3389/fpls.2013.00423

31. Offermann LR, Giangrieco I, Perdue ML, Zuzzi S, Santoro M, Tamburrini M, et al. Elusive structural, functional, and immunological features of Act d 5, the green kiwifruit kiwellin. J Agr Food Chem 2015; 63(29): 6567-76. doi: 10.1021/acs.jafc.5b02159

32. Cavica M, Grozdanovic MM, Bajic A, Jankovic R, Andjus PR, Gavrovic-Jankulovic M. The effect of kiwifruit (Actinidia deliciosa) cysteine protease actinidin on the occludin tight junction network in T84 intestinal epithelial cells. Food Chem Toxicol 2014; 72: 61-8. doi: 10.1016/j.fct.2014.07.012

33. Pastorello EA, Conti A, Pravettoni V, Farioli L, Rivolta F, Ansaloni R, et al. Identification of actinidin as the major allergen of kiwi fruit. J Allergy Clin Immunol 1998; 101(4): 531-7. doi: 10.1016/S0091-6749(98)70360-4

\section{*Tatsuya Moriyama}

Department of Applied Biological Chemistry,

Graduate School of Agriculture, and

Agricultural Technology and Innovation

Research Institute, Kindai University,

3327-204 Naka-machi, Nara 63I-8505, Japan

Email: tmoriyama@nara.kindai.ac.jp 